\title{
Seven COVID-19 Patients Treated with C-Reactive Protein (CRP) Apheresis
}

\author{
Fabrizio Esposito ${ }^{1,}$, , Harald Matthes ${ }^{2,3}$, Friedemann Schad ${ }^{4}$
}

1 Intensiv-Notfallmedizin und Kardiologie, Gemeinschaftskrankenhaus Havelhöhe, Berlin, Germany;

2 Gastroenterologie, Gemeinschaftskrankenhaus Havelhöhe, Berlin, Germany;

3 Medizinischen Klinik für Gastroenterologie, Infektiologie und Rheumatologie and Institut für Sozialmedizin, Epidemiologie und Gesundheitsökonomie, Charité-Universitätsmedizin Berlin Germany;

4 Interdisziplinäre Onkologie und Supportivmedizin, Gemeinschaftskrankenhaus Havelhöhe, Berlin, Germany;

* Correspondence: fabrizio.esposito@havelhoehe.de

\begin{abstract}
Background: The fulminant course of COVID-19, triggered by severe acute respiratory syndrome coronavirus 2 (SARS-CoV-2) presents with a high mortality rate and still lacks a causative treatment. C-reactive protein (CRP) has been shown to increase dramatically during the disease progression and correlates with deleterious outcomes. Selective CRP apheresis can reduce circulating CRP levels fast and effective.

Methods: Seven hospitalized patients with documented severe COVID-19 progression, elevated CRP plasma levels (> $100 \mathrm{mg} / \mathrm{L}$ ) and signs of respiratory failure were treated with CRP apheresis. 2-12 CRP apheresis sessions were performed generally in $24 \mathrm{~h}$ time intervals and depending on CRP plasma levels.
\end{abstract}

Results: CRP apheresis reduced CRP plasma levels by up to $84 \%$ within a few hours, without exhibiting side effects in any patient. Despite signs of severe lung infiltration in all patients, only one patient died. The other patients showed improvements within the chest $\mathrm{x}$-ray after CRP apheresis and were able to recover regardless of intubation and/or ECMO (4 patients). All remaining six patients were discharged from the hospital in good clinical condition. All patients had comorbidities.

Conclusions: This case series presents a mortality rate of only $14 \%$, which is dramatically lower than expected from the presented CRP levels as well as comorbidities and ventilation requirements. Our clinical observations regarding the here presented seven patients support the hypothesis that CRP is a candidate to be therapeutically targeted in the early stage of severe COVID-19.

Keywords: blood component removal; C-reactive protein; CRP apheresis; COVID-19; multiple organ failure; pulmonary fibrosis; SARS virus

\section{Introduction}

C-reactive protein (CRP) is an established biomarker of infection since it was first described in 1930 by Tillet and Francis [1] and can be used as a reliable and fast indicator of the extent of inflammation in the human body. As a classical acute phase protein, CRP rises dramatically within hours of infection or incident and has been shown to activate the complement system via the classical pathway [2] and macrophages via Fc $\gamma$-receptors $[3,4]$. Recently, CRP is not assumed to be only a marker anymore but hypothesized to be an active player in inflammation-induced deleterious tissue processes. This is mainly based on its cytotoxic activity within ischemic and inflamed tissue [5]. After binding to the cell surface, the CRP pentamer may dissociate into monomers suspected to be the pathological agent [6-10]. The question of guilt regarding monomeric or pentameric CRP 
cannot be clarified by this registry study. Based on the description of the function of the CRP adsorber, we can at least conclude that this medical device adsorbs pentameric CRP [11]. We lack the means to investigate whether it breaks down into the monomer after binding to its target structure.

The publication of pathologists [12] has shown that in lung engagement the innate immune system is massively represented but not the adaptive one and also not SARS-CoV-2. In COVID-19 it seems to be the other way as usual. First a lot of adaptive immune system can be detected and thereafter the innate immune system causes damage in the lungs.

In the context of the SARS-CoV-2-induced disease COVID-19 it is remarkable that CRP plasma levels rise to an extent similar to bacterial infections [13]. Further, CRP levels correlate with worse prognosis in COVID-19 with an odds ratio of 18.9 [14] and were proven to be a reliable marker for numerous deleterious processes, as e.g. the need for mechanical ventilation $[13,15]$. Hence, therapeutically targeting CRP was suggested early on during the pandemic $[16,17]$.

CRP apheresis is an extracorporeal procedure, which decreases CRP plasma levels selectively and with no side effects. Thereby, CRP can finally be targeted therapeutically and specifically [18-21]. It was recently introduced as a potential treatment of severe SARS-CoV-2-induced pneumonia [16,22]. After three case reports describing individual healing attempts [22-24] the "C-reactive protein Apheresis in COVID" (CACOV; DRKS00024376) registry was initiated, which already led to the publication of a case series by another participating center [25]. The seven severe COVID-19 patients treated there survived in good health. Further and based on the results of the CACOV registry, the randomized "C-reactive protein Apheresis for Attenuation of Pulmonary, Myocardial and/or Kidney Injury in COVID-19" (CAPMYKCO; NCT04898062) trial was designed.

From the experience with CRP apheresis in myocardial infarction, we concluded that the earliest possible time for the use of CRP apheresis should be aimed for, which we assume to be in the first 72 hours after the onset of severe COVID-19. A publication by Mueller et al. reported that a CRP increase after hospitalization of $13 \mathrm{mg} / \mathrm{l}$ within 48 hours indicates a poor prognosis including invasive ventilation [13]. The same was shown for CRP levels on admission to the hospital. Here, the threshold value is approximately $146 \mathrm{mg} / \mathrm{l}$. Another publication puts the CRP cutoff value at around $97 \mathrm{mg} / 1$ [26]. This report summarizes the treatment of seven COVID-19 patients suffering from severe SARS-CoV-2-induced pneumonia treated by CRP apheresis.

\section{Materials and Methods}

\subsection{CACOV registry}

The CACOV registry is a non-interventional study to investigate the reduction of C-reactive protein (CRP) by selective C-reactive protein apheresis in patients with COVID-19 and highly elevated CRP plasma levels. This analysis includes the first seven patients with severe SARS-CoV-2-induced pneumonia and signs of respiratory failure who exceeded CRP plasma levels of $100 \mathrm{mg} / \mathrm{L}$ and who could be subjected to CRP apheresis in the early phase (first $72 \mathrm{~h}$ ) of severe COVID-19. Patients were recruited between March and May 2021. All patients provided written informed consent. In this registry, the only inclusion criterion is, that the patient should have elevated CRP and be treated with CRP apheresis. All patients required intensive care.

\subsection{CRP apheresis}

A regenerative single adsorbent system was used for CRP apheresis (PentraSorb ${ }^{\circledR}$ CRP; Pentracor GmbH, Hennigsdorf, Germany). Apheresis is performed in cycles, alternating between loading the adsorber with patient plasma and regeneration, which follows a fixed sequence of wash solutions $(\geq 100 \mathrm{~mL} \mathrm{NaCl}, \geq 60 \mathrm{~mL}$ glycine/ $\mathrm{HCl}, \geq 80 \mathrm{~mL}$ PBS, and $\geq 80 \mathrm{~mL} \mathrm{NaCl}$ ). The flow of plasma and wash solutions during apheresis was 
controlled by an automated plasma flow management software module (ADAsorb, medicap clinic $\mathrm{GmbH}$, Ulrichstein, Germany). Blood collection was performed via central venous access because of the high clotting tendency of COVID-19 patients. Plasma separation was performed with the centrifuge (SpectraOptia, TerumoBCT, Denver, CO, USA). For plasma separation, blood was anticoagulated with 1:15 citrate buffer (Anticoagulant Citrate Dextrose Solution A = ACD-A). Plasma flow through the adsorber was 25 to $40 \mathrm{ml} / \mathrm{min}$. Blood flow ranged from 47 to $90 \mathrm{ml} / \mathrm{min}$. Up to $8000 \mathrm{ml}$ of plasma was processed during the treatments, preferably in cycles (change of loading and regeneration of the adsorber) of 1000 and $500 \mathrm{ml}$, respectively. For routine monitoring of apheresis, blood was drawn from the extracorporeal circulation before and after each treatment to determine the CRP concentration.

\subsection{Ventilation scheme}

In hypoxic patients or $\mathrm{SpO} 2<90 \%$, we started oxygen therapy by goggle or mask, high-flow oxygen therapy, and later noninvasive ventilation if necessary, taking into account that delay of intubation in the absence of response to non-invasive ventilation (NIV) worsens the prognosis. In parallel, we performed restrictive fluid therapy in circulatory stable patients.

In invasively ventilated patients, we aimed for a protective ventilation strategy with $\mathrm{Vt} \leq 6 \mathrm{ml} / \mathrm{kg}$, driving pressure $<15 \mathrm{~cm} \mathrm{H} \mathrm{H}_{2} \mathrm{O}$, end-inspiratory airway pressure (pInsp) $<28$ $\mathrm{cm} \mathrm{H}_{2} \mathrm{O}$, and the PEEP setting was based on the so-called high-PEEP table. We performed early abdominal positioning with at least $16 \mathrm{~h}$ abdominal positioning intervals. In case of refractory hypoxemia, inhalative application of nitric oxide (NO) was performed and recruitment maneuvers were considered if necessary after sonography/CT/EIT. In case of persistent hypoxia, after exhaustion of further therapeutic measures, exclusion of contraindications and consultation with relatives regarding the patient's wishes, the use of venovenous extracorporeal membrane oxygenation (ECMO) was performed.

\subsection{Contraindications}

Liver failure, hepatic insufficiency, as citrate is used as an anticoagulant in apheresis.

\subsection{CRP apheresis}

A regenerative single adsorbent system was used for CRP apheresis (PentraSorb ${ }^{\circledR}$ CRP; Pentracor GmbH, Hennigsdorf

\section{Results}

\subsection{Patient characteristics}

We used defined criteria for the selection of patients for this case series. Patients had to be diagnosed with the severe course of COVID-19 and the first CRP apheresis had to be performed a maximum $72 \mathrm{~h}$ after the onset of this severe course. The severe disease course was defined by the requirement for oxygen supply, a CRP plasma concentration $>100 \mathrm{mg} / \mathrm{L}$, a poor overall condition and visible COVID-19 infiltrates in the chest $\mathrm{X}$-ray/CT.

Patient characteristics are summarized in table 1. Age, sex, preexisting and concurrent diseases, hospital length of stay (7-75 d), ventilation therapy (exact type indicated) as well as treatment and apheresis data are shown. All seven patients required either non-invasive oxygen supply or invasive ventilation therapy and suffered from concomitant diseases. Six of seven patients suffered from bacterial superinfection during the hospital stay and therefore received antibiotic therapy.

Patients received 2-12 apheresis sessions depending on their CRP concentration and overall condition. CRP depletion rates ranged from $18-84 \%$ per session, strongly depending on the processed plasma volume, stage of CRP synthesis (acute phase) and initial concentration (see Figure 2 for detailed kinetics).

Only one patient (6) died, all others could be discharged in good clinical condition without the requirement of further ventilation. 


\begin{tabular}{|c|c|c|c|c|c|c|c|c|c|c|c|c|c|c|c|c|}
\hline 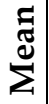 & ڤે. & & 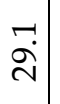 & & & $\stackrel{m}{\stackrel{m}{n}}$ & & & & & & & & & & \\
\hline$\wedge$ & ก) & 工工 & $\approx$ & 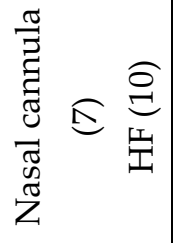 & 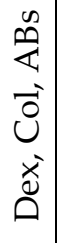 & 10 & 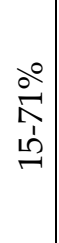 & $\begin{array}{l}1 \\
10 \\
0 \\
0 \\
0\end{array}$ & $\underbrace{\stackrel{\overbrace{}}{0}}_{\lambda}$ & $\lambda$ & & $F$ & $\approx$ & $\lambda$ & & 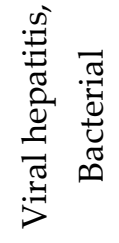 \\
\hline 0 & 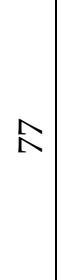 & $\Sigma$ & $\stackrel{m}{-}$ & 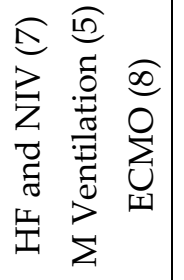 & 党 & $\triangle$ & $\begin{array}{l}\stackrel{0}{\circ} \\
\stackrel{\nabla}{1} \\
\infty \\
-1\end{array}$ & $\overrightarrow{\grave{\Lambda}}$ & 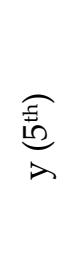 & $\approx$ & & 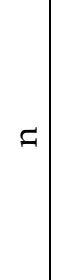 & 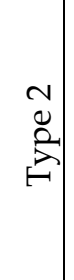 & $\lambda$ & 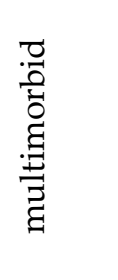 & 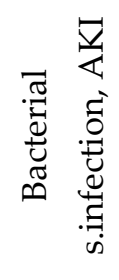 \\
\hline 10 & $\mathbb{N}$ & $\Phi$ & $\sigma$ & 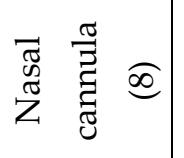 & $\begin{array}{l}\overrightarrow{0} \\
\dot{x} \\
\tilde{0}\end{array}$ & $N$ & $\begin{array}{l}\stackrel{0}{N} \\
\stackrel{1}{\Lambda}\end{array}$ & Ho & $\underbrace{\stackrel{\widetilde{d}}{d}}_{\lambda}$ & $\lambda$ & & $\lambda$ & $=$ & $=$ & 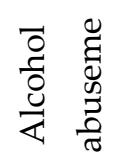 & \\
\hline$F$ & F् & $\Sigma$ & 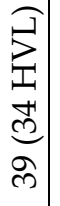 & 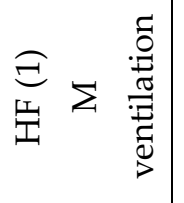 & 党 & $\Lambda$ & 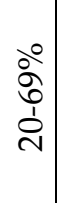 & 옴 & $\underset{\lambda}{\stackrel{\widetilde{d}}{d}}$ & $\therefore$ & & $\lambda$ & 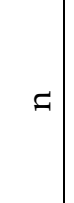 & $\lambda$ & & 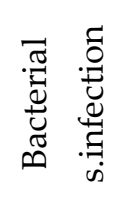 \\
\hline$m$ & aे & $\Sigma$ & $\stackrel{\llcorner}{\wedge}$ & 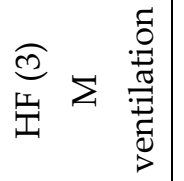 & 党 & 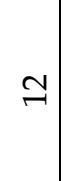 & 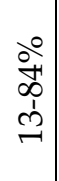 & 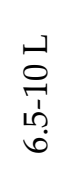 & $\underbrace{\stackrel{\tilde{E}}{E}}_{\lambda}$ & $\lambda$ & & $\lambda$ & 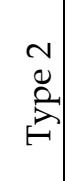 & $\lambda$ & & 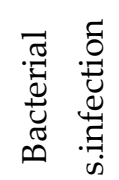 \\
\hline$N$ & ம் & 圷 & $\mathbb{F}$ & 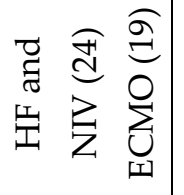 & 党 & $N$ & $\begin{array}{l}\stackrel{0}{2} \\
\stackrel{1}{1} \\
\text { ôे }\end{array}$ & 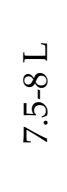 & $\underset{\lambda}{\stackrel{\widehat{E}}{=}}$ & $\lambda$ & & $\lambda$ & 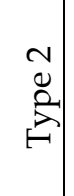 & $\lambda$ & & 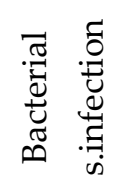 \\
\hline-1 & $\tilde{m}$ & 工 & $\triangle$ & 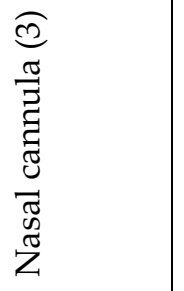 & 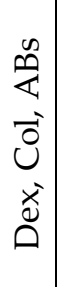 & $N$ & 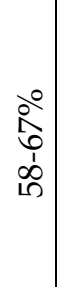 & $\stackrel{\vec{L}}{\stackrel{\sim}{\Lambda}}$ & i & $\lambda$ & & $\lambda$ & $=$ & $\lambda$ & 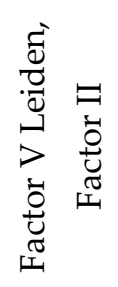 & 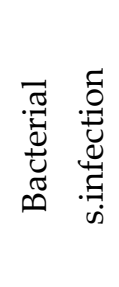 \\
\hline 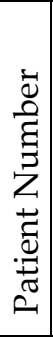 & $\underset{4}{\mathscr{\alpha}}$ & $\begin{array}{l}\stackrel{x}{\varpi ~} \\
\omega\end{array}$ & 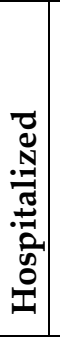 & 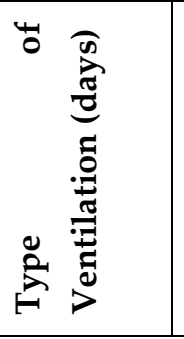 & 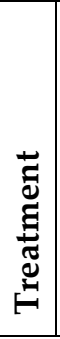 & 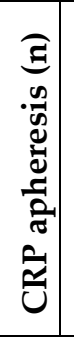 & $\stackrel{\widetilde{u}}{\tilde{u}}$ & 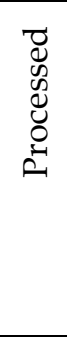 & 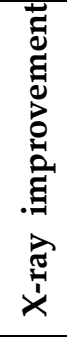 & 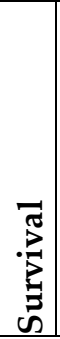 & 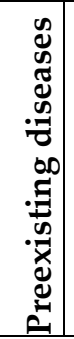 & 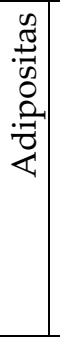 & 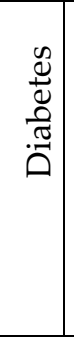 & & 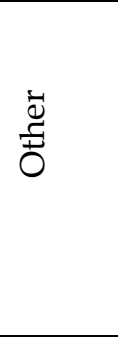 & 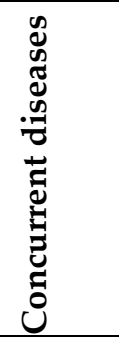 \\
\hline
\end{tabular}


Table 1. Patient characteristics. Table shows age, sex, concomitant diseases, respiratory supply, in-hospital length of stay and treatment parameters of all 7 patients. All patients had documented SARS-CoV-2-induced pneumonia and showed signs of respiratory failure. Patient 6 had acute renal failure (AKI) shortly before his demise. F female, M male, HF High Flow, NIV non-invasive ventilation, $\mathrm{M}$ ventilation mechanical ventilation, y yes, $\mathrm{n}$ no, ECMO extracorporeal membrane oxygenation, Dex Dexamethasone, Col Colchicine, ABs antibiotics, HVL Gemeinschaftskrankenhaus Havelhöhe (our hospital).

\subsection{X-ray / CT chest scans}

All patients received an initial chest x-ray / CT upon hospital admission and subsequent follow-up scans during or after their stay as follow up, depending on ventilation interventions and overall condition. The time between scans varied from 5 to 115 days.

All surviving patients showed improvements within the follow-up x-ray scans after apheresis sessions (Figure 1). Even patient 6 initially showed lung infiltration improvement after the $5^{\text {th }}$ apheresis session, before deteriorating to multi-organ failure and death.

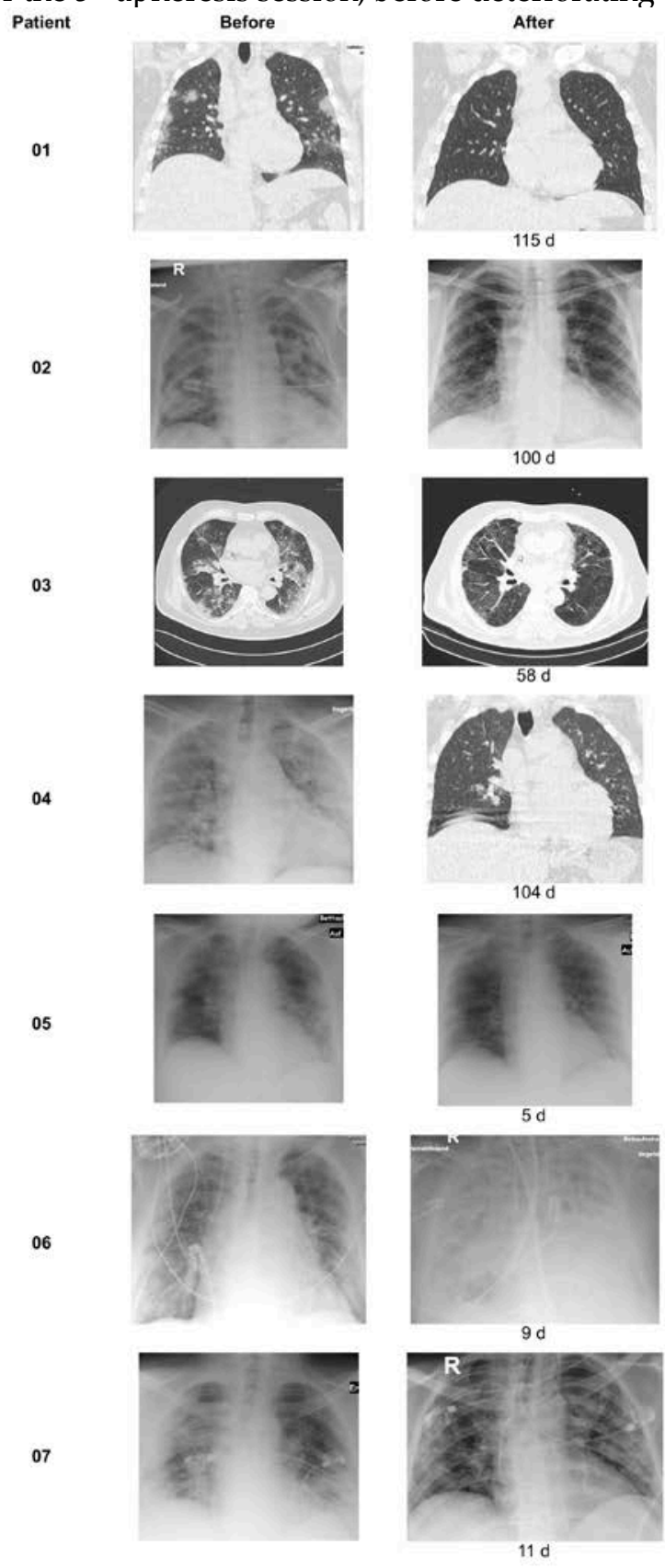

Figure 1. X-ray / CT chest scans. Chest scans were performed before and after treatment / as follow up. The time (days) between the different scans is indicated at the second chest scan. 


\subsection{CRP kinetics}

Figure 2 depicts the CRP plasma kinetics of each patient. CRP levels were elevated (> $100 \mathrm{mg} / \mathrm{L}$ ) on admission in all patients except patients 2 and 6, who then showed rising levels within the first 20-50 hours after admission. CRP apheresis sessions (grey bars) always led to a pronounced decrease in CRP levels. Patients 1, 4, 5 and 7 were treated with aphereses until CRP declined below $100 \mathrm{mg} / \mathrm{L}$ and stayed low. Patients 2 and 3 showed a marked increase in CRP levels after their last apheresis session $(\sim 300-350 \mathrm{~h}$ after admission), which can be correlated with the diagnosed bacterial superinfection (Procalcitonin levels of other patients in supplementary figures 1-6) and was not treated with CRP apheresis but with antibiotics. CRP levels declined before release. CRP levels re-bound steadily after each CRP apheresis session in Patient 6 and could not be maintained below $100 \mathrm{mg} / \mathrm{L}$ until the patient unfortunately died.
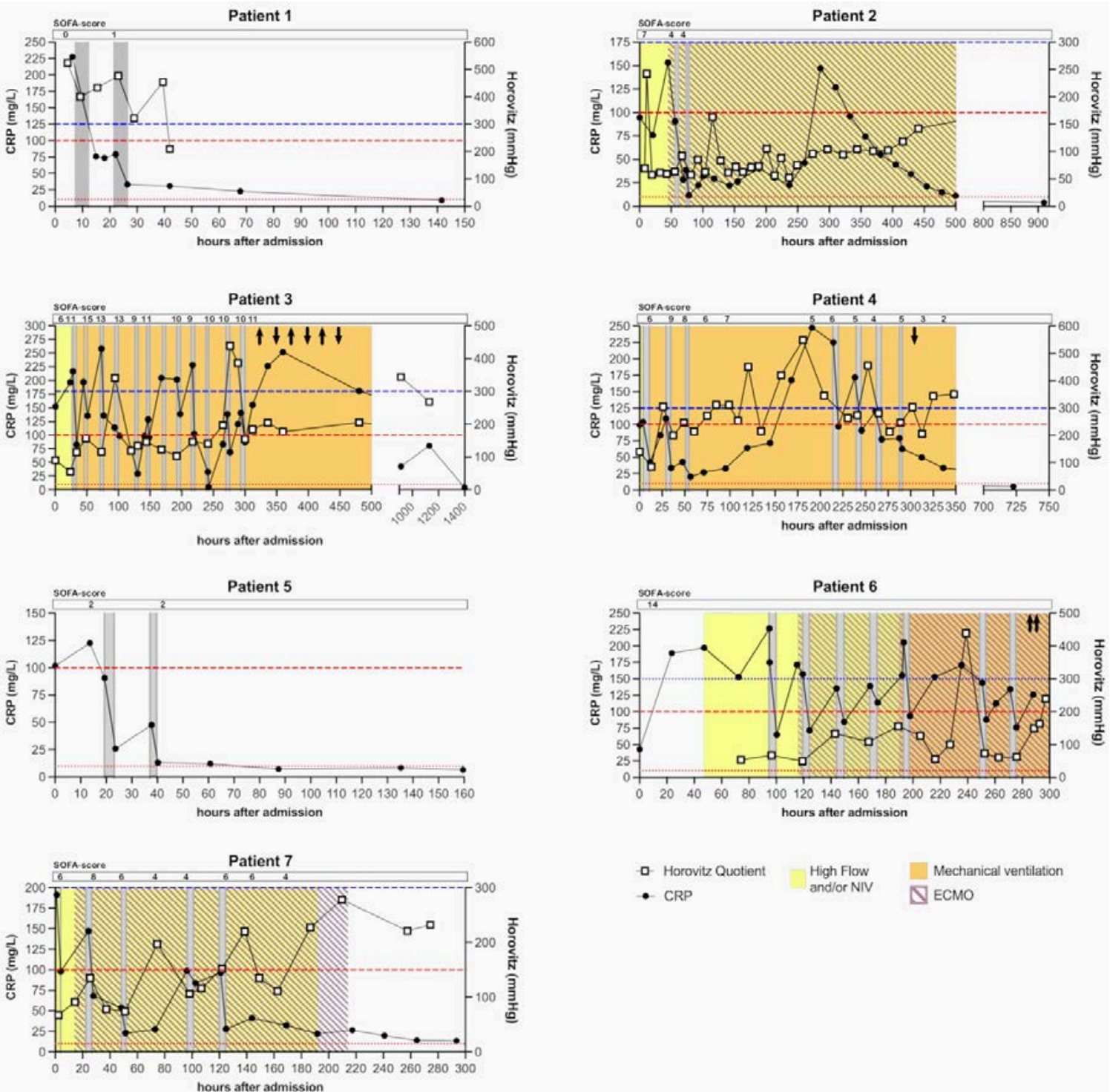

$\begin{array}{lll}\text { - Horovitz Quotient } & \text { High Flow } & \text { Mecha } \\ \text { - CRP } & \text { and/or NIV }\end{array}$

Figure 2. CRP kinetics, Horovitz Quotient and SOFA scores of all patients of the case series. CRP was measured at least every $24 \mathrm{~h}$ in all patients during in-hospital stay. Red lines indicate $10 \mathrm{mg} / \mathrm{L}$ and $100 \mathrm{mg} / \mathrm{L}$ as reference for normal levels and cut-off for severe COVID-19 progression respectively. Blue line indicates $300 \mathrm{mmHg}$ as cut-off for an acute lung injury measured by the Horovitz Quotient. Grey bars indicate apheresis treatments. Ventilation by High Flow or Non-Invasive Ventilation (NIV) is marked in yellow. Mechanical ventilation is marked in orange and extracorporeal membrane oxygenation (ECMO) is marked with purple stripes. No ventilation and nasal cannula are not indicated (for details see table 1). SOFA scores are displayed at corresponding timepoints. For patient 6 the SOFA score was only determined once. 


\subsection{Respiratory and laboratory parameters}

Figure 3 summarizes respiratory parameters in one representative patient (Patient 4 ), figure 3 summarizes other laboratory results of the same patient.

All patients except patient 6 markedly improved their respiratory parameters during hospitalization (Figure 3). Patient 4 specifically showed metabolic alkalosis (elevated $\mathrm{pH}$ and elevated $\left.\mathrm{HCO}_{3}\right)$. He was mechanically ventilated for 24 days $(\sim 150-570 \mathrm{~h}$ after admission) and showed elevated lactate levels, which normalized over time. All patients exhibited signs of respiratory or metabolic alkalosis, which is known to occur in COVID-19 [27].

The other laboratory values (Figure 4) included Procalcitonin, which was elevated in all patients except patient 5 , who solely did not receive antibiotic treatment. Four patients had a laboratory constellation of infections at or shortly after admission. Therefore, they were not treated by immunomodulation and immunosuppression. Other parameters (Ferritin, Fibrinogen, D-Dimers, LDH and CK) were also elevated in numerous patients (including 4) but decreased during the hospital stay and were normal upon discharge. Only patient 6 developed signs of multiple organ failure showing rising levels of Bilirubin, Creatinine, LDH and Procalcitonin as well as a severe acidosis. Patient 6 had a SOFA score of 14 at admission to our ICU.
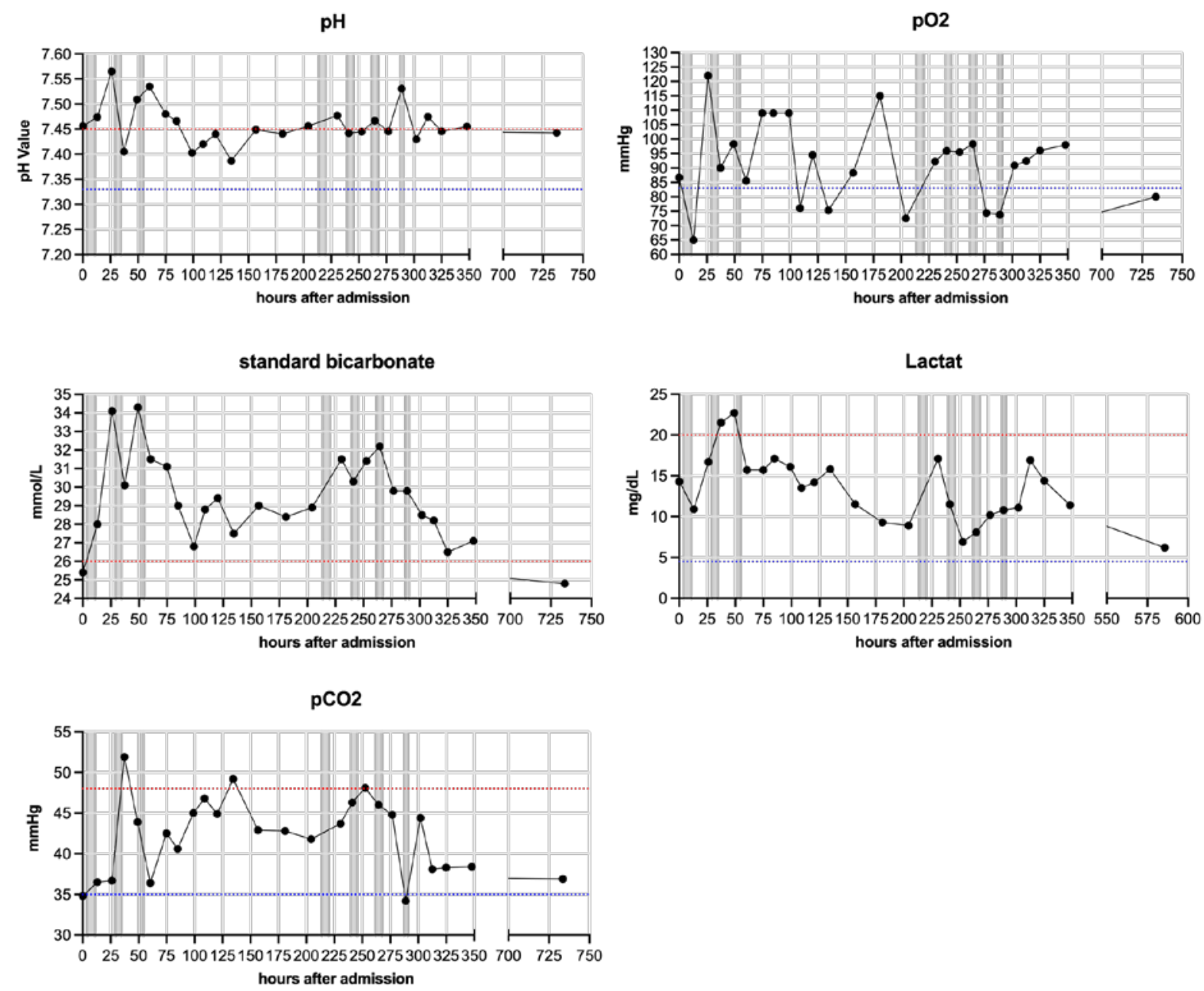

Figure 3. Respiratory parameters of one patient (Patient 4). $\mathrm{pH}$, standard $\mathrm{HCO}_{3}{ }^{-}$, lactate, arterial $\mathrm{pCO}_{2}$ and $\mathrm{pO}_{2}$ were measured regularly for each patient and are depicted here representatively for patient 4 . Blue lines indicate minimum baseline and red lines maximum baseline for each normal range. Grey bars indicate apheresis treatments. 

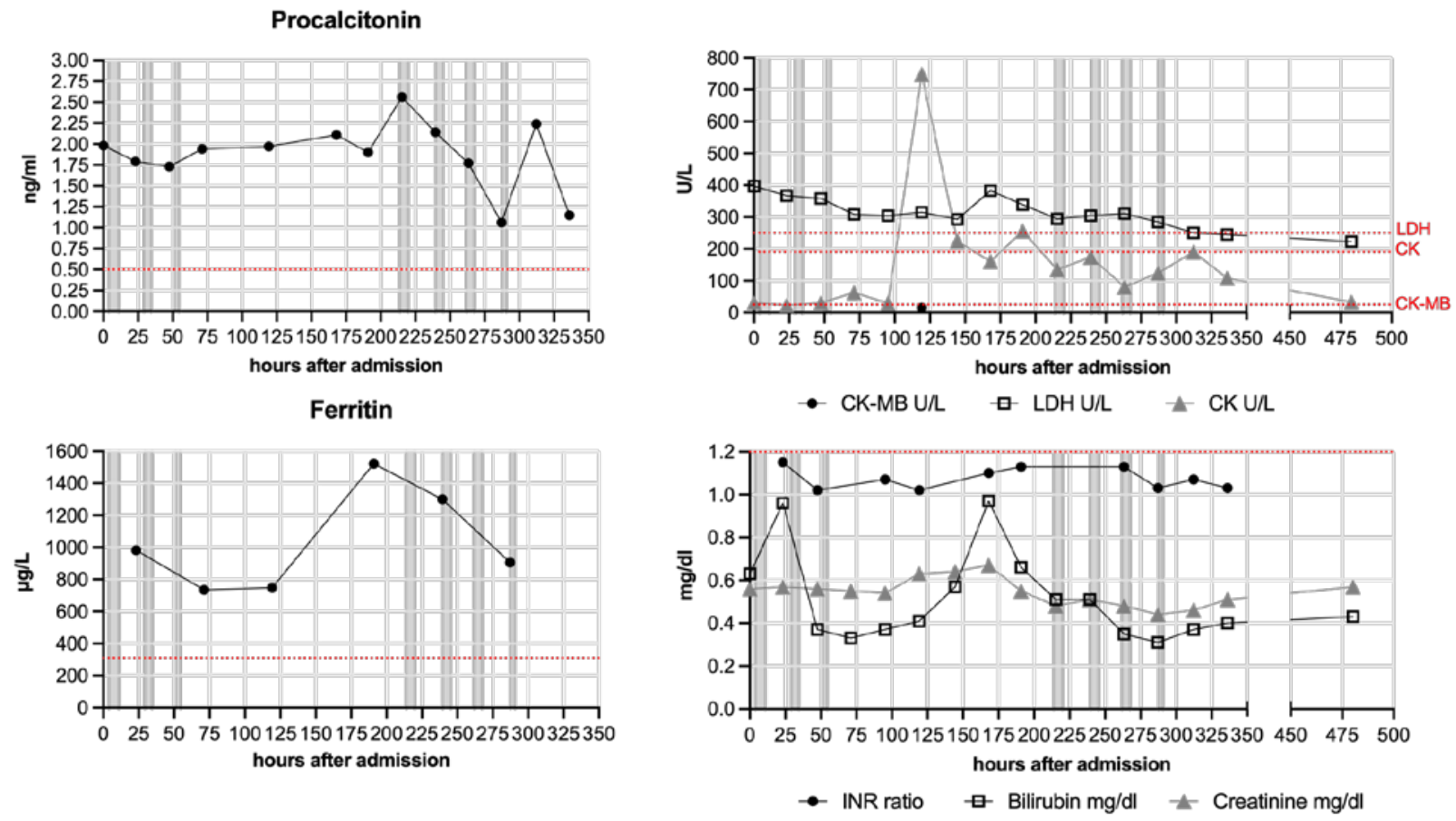

Fibrinogen
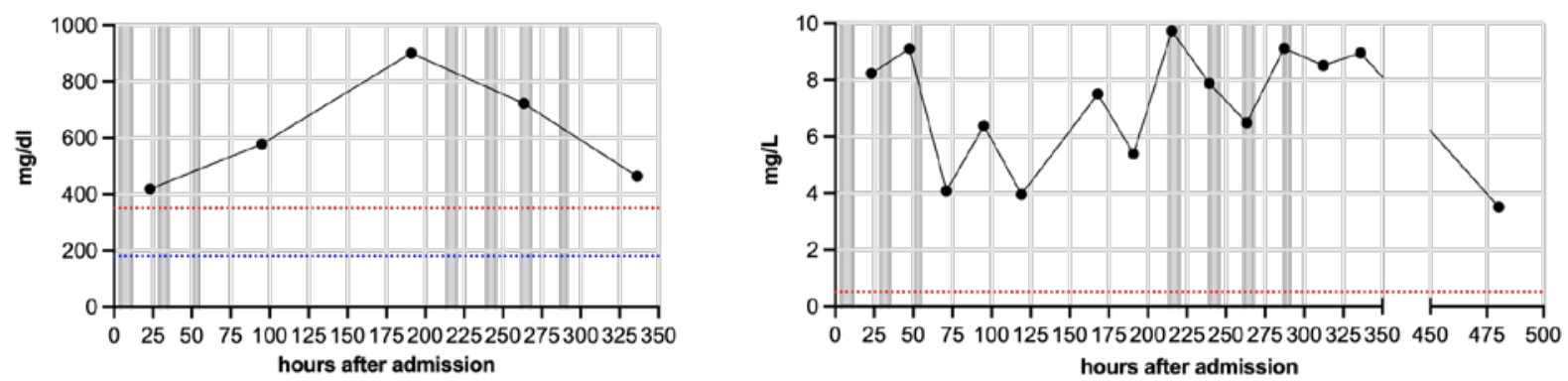

Figure 4. Other laboratory parameters of one patient (Patient 4). Procalcitonin, CK-MB, CK, LDH, Ferritin, INR ratio, Bilirubin, Creatinine, Fibrinogen and D-Dimers were measured regularly for each patient and are depicted here representatively for Patient 4 . Blue lines indicate minimum baseline and red lines maximum baseline for each normal range. Grey bars indicate apheresis treatments.

\subsection{Control cohort}

At the start of the CACOV registry, we had treated 30 severe COVID-19 patients according to the respectively valid guidelines. Of this cohort, 3 developed $C R P<100$ $\mathrm{mg} / \mathrm{l}$. All of them survived. The other 27 developed CRP $>100 \mathrm{mg} / \mathrm{l}$. Thirteen of them died which is $48 \%$.

\section{Discussion}

The severe course of COVID-19, with its high mortality rate, is fundamentally caused by an excessive immune response, often-called cytokine storm, which mediates the destruction of mainly pulmonary tissue $[28,29]$. CRP has been established as one of the key effector molecules of this process $[5,15,30]$. Reducing its circulating levels is the next logical step in order to inhibit the deleterious destruction of lung cells, which could recover during ventilation and with more time. CRP apheresis presents the first therapeutic opportunity to target CRP selectively and quickly.

Three COVID-19 patients have been treated with CRP apheresis as individual healing attempts before [22-24]. Subsequently, the CACOV registry started and is running at several centers throughout Germany. Here, we publish a case series of seven patients, with the following characteristics. 
All patients reported here were diagnosed with the severe course of COVID-19 and CRP apheresis was initiated within $72 \mathrm{~h}$ of this onset. Further, CRP serum levels exceeded $100 \mathrm{mg} / \mathrm{L}$. CRP apheresis significantly decreased the elevated CRP plasma levels in all seven patients, which supports the efficacy of this therapy and is in line with all patients treated with CRP apheresis so far [19-24].

Apart from one patient (6), who unfortunately died of organ failure 14 days after hospital admission, all patients recovered from COVID-19 and could be discharged in good clinical condition after 2-12 CRP apheresis sessions.

All patients besides patient 5 showed elevated Procalcitonin levels and had to be treated with antibiotics. Further, three patients received Dexamethasone/Colchicine upon hospital admission (table 1), which is known to lower CRP plasma levels to a certain extent for a short period. However, this was not robustly visible within the individual CRP kinetics (figure 1) and the performed CRP apheresis, with an up to $84 \%$ reduction within hours, was definitely more efficient in decreasing CRP plasma levels.

Based on the existing literature and our results, we assume that fluid retention in the lungs and the hypoxia induced by it provide for the induction of CRP (Sheriff et al., 2021, Ringel et al., 2021). The CRP kinetics then depends on the kinetics of the fluid retention and also on the kinetics of the disappearance of the fluid.

Focusing on the CRP kinetics, all patients depicted maximum CRP levels over 120 $\mathrm{mg} / \mathrm{L}$. The significant correlation of the increase in CRP as well as the maximum CRP amount with mortality has been widely established so far [14,31-33]. In detail, in one study a $108 \mathrm{mg} / \mathrm{L}$ CRP cut-off led to a higher mortality of $32 \%$ vs $18 \%$ [15]. In another report, a maximum CRP serum concentration of $>100 \mathrm{mg} / \mathrm{L}$ was associated with either progressive or severe COVID-19 with a mortality of up to $59 \%$ vs $4 \%$ in the mild group (< $100 \mathrm{mg} / \mathrm{L}$ ) [13]. In our in house control cohort, 48\% of severe COVID-19 patients which developed CRP > $100 \mathrm{mg} / \mathrm{l}$ died. However, in our case series the mortality rate was only $14 \%$ and thereby dramatically lower than expected from the presented CRP levels as well as co-morbidities and ventilation requirements. All of them are in good health in February 2022 and therefore at least 9 months after discharge from the hospital. This remarkable, as reports from Germany [34] and the US [35] showed that there is a significantly increased risk for mortality over the next 6 or 12 months, respectively. Our clinical observations regarding the here presented seven patients support the hypothesis that CRP should be therapeutically targeted in COVID-19.

\section{Study Limitations}

In order to conclusively proof that CRP apheresis is the therapy of choice in severe COVID-19 courses, randomized controlled trials are urgently needed.

Author Contributions: FE acquired and analyzed the data and wrote the manuscript. HM and FS edited the manuscript. All authors contributed to the article and approved the submitted version.

Funding: The CACOV-registry is supported by Pentracor GmbH, Hennigsdorf, Germany.

Institutional Review Board Statement: The studies involving human participants were reviewed and approved by Ethik-Kommission der Ärztekammer Berlin No. 28/21. All patients gave their written informed consent.

Data Availability Statement: The original contributions presented in the study are included in the article/supplementary material, further inquiries can be directed to the corresponding author.

Conflicts of Interest: These patients were treated within the CACOV registry. The manufacturer Pentracor $\mathrm{GmbH}$ is supposed to pay a fee as agreed with the ethics committee for the effort to complete the CRFs. The authors declare that the research was conducted in the absence of any other commercial or financial relationships that could be construed as a potential conflict of interest. 
Acknowledgments: We gratefully acknowledge Anja Ramlow and Charlene Edge, Pentracor $\mathrm{GmbH}$, Germany, for organizing and performing CRP apheresis

\section{References}

1. Tillett, W.S.; Francis, T. Serological Reactions in Pneumonia with a Non-Protein Somatic Fraction of Pneumococcus. J Exp Med 1930, 52, 561-571.

2. Kaplan, M.H.; Volanakis, J.E. Interaction of C-reactive protein complexes with the complement system. I. Consumption of human complement associated with the reaction of C-reactive protein with pneumococcal C-polysaccharide and with the choline phosphatides, lecithin and sphingomyelin. Journal of immunology 1974, 112, 2135-2147.

3. Bharadwaj, D.; Stein, M.P.; Volzer, M.; Mold, C.; Du Clos, T.W. The major receptor for C-reactive protein on leukocytes is fcgamma receptor II. J Exp Med 1999, 190, 585-590.

4. Manolov, D.E.; Rocker, C.; Hombach, V.; Nienhaus, G.U.; Torzewski, J. Ultrasensitive confocal fluorescence microscopy of C-reactive protein interacting with FcgammaRIIa. Arteriosclerosis, thrombosis, and vascular biology 2004, 24, 2372-2377, doi:10.1161/01.ATV.0000147407.17137.02.

5. Sheriff, A.; Kayser, S.; Brunner, P.; Vogt, B. C-Reactive Protein Triggers Cell Death in Ischemic Cells. Front Immunol 2021, 12, doi:10.3389/fimmu.2021.630430.

6. Boncler, M.; Wu, Y.; Watala, C. The Multiple Faces of C-Reactive Protein-Physiological and Pathophysiological Implications in Cardiovascular Disease. Molecules (Basel, Switzerland) 2019, 24, doi:10.3390/molecules24112062.

7. Braig, D.; Nero, T.L.; Koch, H.-G.; Kaiser, B.; Wang, X.; Thiele, J.R.; Morton, C.J.; Zeller, J.; Kiefer, J.; Potempa, L.A.; et al. Transitional changes in the CRP structure lead to the exposure of proinflammatory binding sites. Nature Communications 2017, 8, 14188, doi:10.1038/ncomms14188.

8. McFadyen, J.D.; Kiefer, J.; Braig, D.; Loseff-Silver, J.; Potempa, L.A.; Eisenhardt, S.U.; Peter, K. Dissociation of C-Reactive Protein Localizes and Amplifies Inflammation: Evidence for a Direct Biological Role of C-Reactive Protein and Its Conformational Changes. Frontiers in Immunology 2018, 9, 1351.

9. Thiele, J.R.; Zeller, J.; Bannasch, H.; Stark, G.B.; Peter, K.; Eisenhardt, S.U. Targeting C-Reactive Protein in Inflammatory Disease by Preventing Conformational Changes. Mediators of Inflammation 2015, 2015, 372432, doi:10.1155/2015/372432.

10. Sproston, N.R.; Ashworth, J.J. Role of C-Reactive Protein at Sites of Inflammation and Infection. Frontiers in immunology 2018, 9, 754-754, doi:10.3389/fimmu.2018.00754.

11. Mattecka, S.; Brunner, P.; Hähnel, B.; Kunze, R.; Vogt, B.; Sheriff, A. PentraSorb C-Reactive Protein: Characterization of the Selective C-Reactive Protein Adsorber Resin. Therapeutic Apheresis and Dialysis 2019, 23, 474-481, doi:10.1111/1744-9987.12796.

12. Nienhold, R.; Ciani, Y.; Koelzer, V.H.; Tzankov, A.; Haslbauer, J.D.; Menter, T.; Schwab, N.; Henkel, M.; Frank, A.; Zsikla, V.; et al. Two distinct immunopathological profiles in autopsy lungs of COVID-19. Nature Communications 2020, 11, 5086, doi:10.1038/s41467-020-18854-2.

13. Mueller, A.A.; Tamura, T.; Crowley, C.P.; DeGrado, J.R.; Haider, H.; Jezmir, J.L.; Keras, G.; Penn, E.H.; Massaro, A.F.; Kim, E.Y. Inflammatory Biomarker Trends Predict Respiratory Decline in COVID-19 Patients. Cell reports. Medicine 2020, 1, 100144, doi:10.1016/j.xcrm.2020.100144.

14. Parimoo, A.; Biswas, A.; Baitha, U.; Gupta, G.; Pandey, S.; Ranjan, P.; Gupta, V.; Barman Roy, D.; Prakash, B.; Wig, N. Dynamics of Inflammatory Markers in Predicting Mortality in COVID-19. Cureus 2021, 13, e19080, doi:10.7759/cureus.19080.

15. Smilowitz, N.R.; Kunichoff, D.; Garshick, M.; Shah, B.; Pillinger, M.; Hochman, J.S.; Berger, J.S. C-reactive protein and clinical outcomes in patients with COVID-19. European heart journal 2021, 42, 2270-2279, doi:10.1093/eurheartj/ehaa1103. 
16. Kayser, S.; Kunze, R.; Sheriff, A. Selective C-reactive protein apheresis for Covid-19 patients suffering from organ damage. Therapeutic apheresis and dialysis : official peer-reviewed journal of the International Society for Apheresis, the Japanese Society for Apheresis, the Japanese Society for Dialysis Therapy 2021, 25, 251-252, doi:10.1111/1744-9987.13532.

17. Pepys, M.B. C-reactive protein predicts outcome in COVID-19: is it also a therapeutic target? European heart journal 2021, 42, 2280-2283, doi:10.1093/eurheartj/ehab169.

18. Sheriff, A.; Schindler, R.; Vogt, B.; Abdel-Aty, H.; Unger, J.K.; Bock, C.; Gebauer, F.; Slagman, A.; Jerichow, T.; Mans, D.; et al. Selective apheresis of C-reactive protein: a new therapeutic option in myocardial infarction? J Clin Apher 2015, 30, 15-21, doi:10.1002/jca.21344.

19. Ries, W.; Sheriff, A.; Heigl, F.; Zimmermann, O.; Garlichs, C.D.; Torzewski, J. "First in Man": Case Report of Selective C-Reactive Protein Apheresis in a Patient with Acute ST Segment Elevation Myocardial Infarction. Case Rep Cardiol 2018, 2018, 4767105, doi:10.1155/2018/4767105.

20. Ries, W.; Heigl, F.; Garlichs, C.; Sheriff, A.; Torzewski, J. Selective C-Reactive Protein-Apheresis in Patients. Therapeutic apheresis and dialysis : official peer-reviewed journal of the International Society for Apheresis, the Japanese Society for Apheresis, the Japanese Society for Dialysis Therapy 2019, 23, 570-574, doi:10.1111/1744-9987.12804.

21. Ries, W.; Torzewski, J.; Heigl, F.; Pfluecke, C.; Kelle, S.; Darius, H.; Ince, H.; Mitzner, S.; Nordbeck, P.; Butter, C.; et al. C-Reactive Protein Apheresis as Anti-inflammatory Therapy in Acute Myocardial Infarction: Results of the CAMI-1 Study. Front Cardiovasc Med 2021, 8, doi:10.3389/fcvm.2021.591714.

Torzweski, J.; Heigl, F.; Zimmermann, O.; Wagner, F.; Schumann, C.; Hettich, R.; Bock, C.; Kayser, S.; Sheriff, A. First-in-man: Case report of Selective C-reactive Protein Apheresis in a Patient with SARS-CoV-2 Infection. The American journal of case reports 2020, 21, e925020, doi:10.12659/AJCR.925020.

23. Ringel, J.; Ramlow, A.; Bock, C.; Sheriff, A. Case Report: C-Reactive Protein Apheresis in a Patient With COVID-19 and Fulminant CRP Increase. Front Immunol 2021, 12, doi:10.3389/fimmu.2021.708101.

24. Torzewski, J.; Zimmermann, O.; Kayser, S.; Heigl, F.; Wagner, F.; Sheriff, A.; Schumann, C. Successful Treatment of a 39-Year-Old COVID-19 Patient with Respiratory Failure by Selective C-Reactive Protein Apheresis. The American journal of case reports 2021, 22, e932964, doi:10.12659/ajcr.932964.

25. Schumann, C.; Heigl, F.; Rohrbach, I.J.; Sheriff, A.; Wagner, L.; Wagner, F.; Torzewski, J. A Report on the First 7 Sequential Patients Treated Within the C-Reactive Protein Apheresis in COVID (CACOV) Registry. The American journal of case reports 2022, 23, e935263, doi:10.12659/AJCR.935263.

26. Herold, T.; Jurinovic, V.; Arnreich, C.; Lipworth, B.J.; Hellmuth, J.C.; von Bergwelt-Baildon, M.; Klein, M.; Weinberger, T. Elevated levels of IL-6 and CRP predict the need for mechanical ventilation in COVID-19. J Allergy Clin Immunol 2020, 146, 128-136 e124, doi:10.1016/j.jaci.2020.05.008.

27. Wu, C.; Wang, G.; Zhang, Q.; Yu, B.; Lv, J.; Zhang, S.; Wu, G.; Wu, S.; Zhong, Y. Association Between Respiratory Alkalosis and the Prognosis of COVID-19 Patients. Front Med 2021, 8, doi:10.3389/fmed.2021.564635.

28. Cappanera, S.; Palumbo, M.; Kwan, S.H.; Priante, G.; Martella, L.A.; Saraca, L.M.; Sicari, F.; Vernelli, C.; Di Giuli, C.; Andreani, P.; et al. When Does the Cytokine Storm Begin in COVID-19 Patients? A Quick Score to Recognize It. Journal of clinical medicine 2021, 10, doi:10.3390/jcm10020297.

29. Nadeem, R.; Elhoufi, A.M.; Iqbal, N.E.; Obaida, Z.A.; Elgohary, D.M.; Singh, M.K.; Zoraey, S.F.; Abdalla, R.M.; Eltayeb, S.Y.; Danthi, C.S.; et al. Prediction of Cytokine Storm and Mortality in Patients with COVID-19 Admitted to ICU: Do Markers Tell the Story? Dubai Medical Journal 2021, doi:10.1159/000514406.

30. Mosquera-Sulbaran, J.A.; Pedreañez, A.; Carrero, Y.; Callejas, D. C-reactive protein as an effector molecule in Covid-19 pathogenesis. Reviews in Medical Virology 2021, n/a, e2221, doi:10.1002/rmv.2221. 
31. Ali, N. Elevated level of C-reactive protein may be an early marker to predict risk for severity of COVID-19. Journal of Medical Virology 2020, 92, 2409-2411, doi:https://doi.org/10.1002/jmv.26097.

32. Sharifpour, M.; Rangaraju, S.; Liu, M.; Alabyad, D.; Nahab, F.B.; Creel-Bulos, C.M.; Jabaley, C.S.; Emory, C.-Q.; Clinical Research, C. C-Reactive protein as a prognostic indicator in hospitalized patients with COVID-19. PLoS One 2020, 15, e0242400, doi:10.1371/journal.pone.0242400.

33. Villoteau, A.; Asfar, M.; Otekpo, M.; Loison, J.; Gautier, J.; Annweiler, C.; group, G.-C.s. Elevated C-reactive protein in early COVID-19 predicts worse survival among hospitalized geriatric patients. PLoS One 2021, 16, e0256931, doi:10.1371/journal.pone.0256931.

34. Günster, C.; Busse, R.; Spoden, M.; Rombey, T.; Schillinger, G.; Hoffmann, W.; Weber-Carstens, S.; Schuppert, A.; Karagiannidis, C. 6-month mortality and readmissions of hospitalized COVID-19 patients: A nationwide cohort study of 8,679 patients in Germany. PLOS ONE 2021, 16, e0255427, doi:10.1371/journal.pone.0255427.

35. Mainous, A.G., 3rd; Rooks, B.J.; Wu, V.; Orlando, F.A. COVID-19 Post-acute Sequelae Among Adults: 12 Month Mortality Risk. Front Med (Lausanne) 2021, 8, 778434, doi:10.3389/fmed.2021.778434. 